\title{
Oncogene SIS
}

National Cancer Institute

\section{Source}

National Cancer Institute. Oncogene SIS. NCI Thesaurus. Code C17135.

Human Oncogene SIS is a mutated variant of PDGFB Gene (PDGF/VEGF Family), which encodes 2 alternative isoforms of 241-aa 27-kDa (precursor) Platelet-Derived Growth Factor-Beta Chain, a potent mitogenic factor for mesenchymal cells characterized by a motif of eight cysteines and released by platelets upon wounding to stimulate adjacent cell growth; PDGF activates a RAS/PIK3/AKT1/IKK/NFKB1 pathway. PDGFB can exist as a disulfide-linked homodimer or heterodimer with Platelet-Derived Growth Factor Alpha. $A A, B B$, and $A B$, dimers bind to PDGF receptor and elicit a variety of cellular responses. $A$ and $B$ homodimers are implicated in transformation. PDGFB mutations are associated with meningioma. Reciprocal $t(17 ; 22)(q 22 ; q 13)$ translocations with COL1A1 result in unregulated growth factor expression and are associated with DFSP. Oncogene SIS disrupts normal cell function. 